\title{
Rhodanese isozymes in three subjects with Leber's optic neuropathy
}

\author{
D B WHITEHOUSE*, C J M POOLE $\dagger$, P R N KIND \\ AND D A HOPKINSON* \\ From * the MRC Human Biochemical Genetics Unit, The Galton Laboratory, University College London, \\ Wolfson House, London NW1 2HE; the Medical Unit, Westminster Hospital, London SW1P 2AP; and \\ $\ddagger$ the Department of Chemical Pathology, St Thomas's Hospital, London SE1 7EH.
}

SUMMARY Previous studies have reported an association between Leber's optic neuropathy and deficiency of rhodanese activity in liver and rectal mucosa. We have studied the rhodanese isozymes in liver biopsies from three subjects with Leber's optic neuropathy. The rhodanese isozyme patterns were indistinguishable from controls both in relative intensity and position on the isoelectric focusing $\mathrm{pH}$ gradient. No new rhodanese isozymes were observed and there was no evidence of deficiency in any of the cases.

Leber's optic neuropathy ${ }^{1}$ is a rare disorder which occurs predominantly in young adult males giving rise to progressive bilateral visual failure. The disease is familial but the pattern of inheritance has not been clearly defined. ${ }^{2}$ In those cases where a family history can be identified the disorder appears to have been transmitted from the mother. Various hypotheses have been proposed to account for the pathology including maternal transmission of an infectious agent, such as a slow virus or a mitochondrial abnormality. ${ }^{3-5}$ Another suggestion has been that the optic atrophy is part of a diffuse neurological disorder resulting from an inborn error of cyanide metabolism. ${ }^{6}$ This hypothesis is attractive since the main route of cyanide detoxication is via the mitochondrial enzyme thiosulphate sulphur transferase (E.C.2.8.1.1.), also known as rhodanese, and a deficiency of this mitochondrial protein might account for both the pathology and the matroclinal pattern of inheritance.

Early studies of Wilson ${ }^{6}$ on rhodanese from two patients with Leber's optic neuropathy failed to confirm the hypothesis since similar levels of activity were found in necropsy specimens of liver from patients and controls. Also, rhodanese assay on a liver biopsy, taken six months before the death of one of these patients, showed no evidence of deficiency. However, in a more recent study Cagianut et $\mathrm{al}^{7}$ reported significant deficiency, about $20 \%$ of the normal level, of rhodanese enzyme activity in liver biopsies from two affected

Received for publication 10 June 1988.

Accepted for publication 19 August 1988. males of a family with a four generation history of Leber's optic neuropathy, thus reviving the hypothesis of an inborn error of cyanide detoxication in this condition. Conflicting results have been obtained in subsequent studies: normal levels of rhodanese activity were found ${ }^{8}$ in tibialis anterior skeletal muscle biopsies from 13 Finnish patients, but a dramatic six-fold reduction in rhodanese activity was discovered $^{9}$ in rectal mucosa biopsies from 10 affected British subjects from nine different families.

It is difficult to reconcile all of these quantitative data on rhodanese activity in patients with Leber's optic neuropathy. One possibility is that the condition is very heterogeneous and the discrepant findings reflect chance selection of patients from different families with or without an underlying disorder of rhodanese. Another interpretation is that the discrepancies are attributable to tissue specific differences in rhodanese isozyme expression since healthy human tissues exhibit very complex patterns of rhodanese isozymes. ${ }^{10}$ There are two main sets of components which are probably coded by separate genes: an acidic cytosolic group of about three isozymes with a universal tissue distribution, including human red cells, and a more complex basic group with heterogeneous tissue distribution but especially prominent in liver and kidney, which may display as many as 14 different components by isoelectric focusing. ${ }^{10}$ On simple quantitative analysis a deficiency of one of these isozymes might manifest in some tissues but not in others from patients with Leber's optic neuropathy and thus account for the heterogeneity in the data obtained by this approach. 
In an attempt to resolve this question we have studied the rhodanese isozyme patterns in liver biopsies from three subjects with Leber's optic neuropathy.

\section{Materials and methods}

Three white men from different families living in southern England were studied. All three subjects had subacute visual loss with bilateral optic atrophy and central scotoma; they were thought to be representative of patients with Leber's optic neuropathy. The mean age of the three subjects was 28 (25 to 30) years and the duration of optic atrophy varied from one to 10 years.

\section{SUBJECT 1}

This man lost vision at the age of 22 years, first in one eye, then two or three weeks later in the other; visual acuity in each eye was reduced to counting fingers. An older brother had similar visual loss in his twenties and there was a partially sighted uncle on his mother's side of the family. He was drinking up to 10 pints of beer a week at the time of liver biopsy.

\section{SUBJECT 2}

This man had similar visual failure to subject 1 when aged 18 years. However, at the age of 20 his vision spontaneously improved to its present visual acuity of 6/9 in each eye. There are two similarly affected brothers and a maternal uncle who went blind at the age of 20 . He was drinking up to 30 pints of beer week at the time of liver biopsy.

SUBJECT 3

This man progressively lost vision over the course of four months to $2 / 60$ visual acuity in each eye. There was no family history of visual failure; howeve fluorescein retinal angiography showed telangiectatic changes in the peripapillary nerve fibre layer charac: teristic of Leber's optic neuropathy. He was drinking up to 15 pints of beer a week at the time of live? biopsy.

Liver biopsy was performed by a standard tech nique; the specimens were transported to the labor atory in dry ice and stored at $-70^{\circ} \mathrm{C}$. Control livet specimens were obtained from routine necrops $\vec{x}$ within 24 hours of sudden deaths from myocardiap infarction, as well as the residues of liver biopsies from patients undergoing diagnostic examination form suspected liver disease. The mean age of the controls was 59 years. Care was taken to avoid repeated freezing and thawing of the samples. The control and test material was treated identically and was coded so that the evaluation of results of the् analyses would be without bias. Rhodanese enzyme assays were carried out as described previously ${ }^{9}$ an isoelectric focusing was performed using Ampholine (LKB) in $\mathrm{pH} 3.5$ to 11 gradients in $5 \%$ polyacryl amide gels and a specific isozyme staining system. $\frac{1 \mathrm{~S}}{\varnothing}$

\section{Results}

The figure shows the rhodanese isozyme patterns

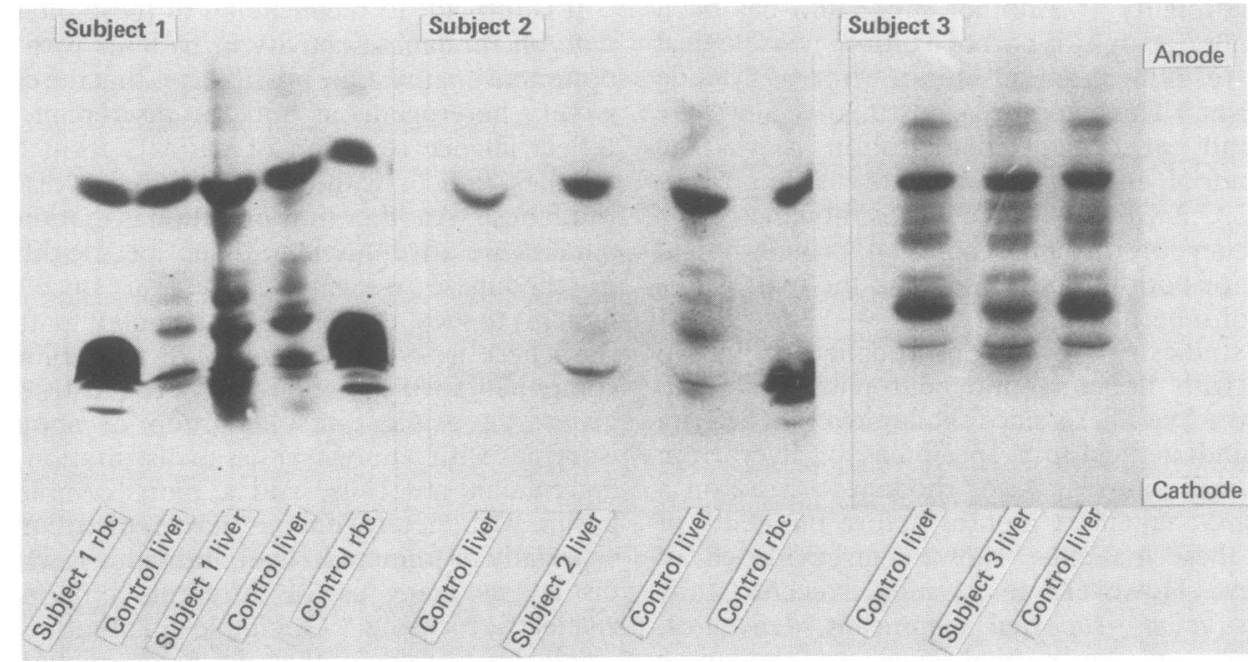

FIGURE Rhodanese isozymes after isoelectric focusing (pH3.5 to 11.0) of liver biopsy specimens from three subjects with Leber's optic neuropathy and six control subjects. The haemolysate (rbc) rhodanese isozyme pattern is also shown for subject 1 and controls; in these tracks the very dark component towards the cathodal side of the pH gradient is haemoglobirk 
obtained after ultrathin polyacrylamide gel isoelectric focusing of homogenates prepared from the liver biopsies of the three subjects with Leber's optic neuropathy. In each case the patient track is flanked by two control liver specimens. Normal rhodanese isozyme patterns were observed in each patient, indistinguishable from the controls both in terms of the relative intensity and total number of isozymes. In each case complex patterns are observed and there is no evidence of reduced intensity or absence of any of the rhodanese bands or additional new isozymes. In subject 2 the photographic reproduction is poor and very detailed analysis is impossible, but in subjects 1 and 3 at least 12 distinct components can be resolved in the patient material and in the control specimens. Furthermore, the overall staining intensity appeared to be at least as high if not higher in these cases than in the controls.

This result was confirmed by direct measurement of the total rhodanese activity in homogenates prepared from the same liver biopsies. Similar high levels, $23467 \mu \mathrm{mol}$ and $19649 \mu \mathrm{mol}$ thiocyanate/g protein/minute respectively, were obtained for subjects 1 and 3 compared to a mean level of $6410 \mu \mathrm{mol}$ for the control necropsy livers $(n=4$, range 3361 to $8242 \mu \mathrm{mol}$ ) and $14656 \mu \mathrm{mol}$ for the control biopsy specimens $(n=5$, range 8212 to $20116 \mu \mathrm{mol})$. Subject 2, which gave the poorest photograph (figure), showed a relatively very high figure of $54924 \mu \mathrm{mol}$.

Blood samples were obtained from each patient and examination by isoelectric focusing showed normal red cell rhodanese isozyme patterns (the figure illustrates the result in subject 1) with no evidence of deficiency or abnormal isoelectric mobility.

\section{Discussion}

We have obtained unequivocal evidence of normal rhodanese isozyme patterns in three unrelated subjects with Leber's optic neuropathy. The isozymes in these subjects were indistinguishable from the controls, both in their relative intensities and in their positions on the isoelectric focusing $\mathrm{pH}$ gradient. No new isozymes were observed.

In all three subjects the rhodanese isozyme patterns comprised 'red cell' and 'tissue' isozymes. The former are cytosolic and were not expected to show any peculiar features, especially since the erythrocyte rhodanese isozyme patterns in these subjects were normal. The 'tissue' rhodanese isozymes are more complex and since some are probably associated with mitochondria a detailed scrutiny of these components was performed, but no anomaly was recorded.

The results and conclusions obtained by the isoelectric focusing experiments were supported by the quantitative rhodanese assays. No evidence for deficiency of total hepatic rhodanese activity was encountered in any of the three Leber's optic neuropathy subjects. Indeed quite high levels of activity were recorded, which could reflect the high daily alcohol intake of these subjects, since similarly high levels were observed in the control liver biopsies obtained from patients with histological evidence of alcoholic liver damage.

In conclusion we have found no evidence of quantitative or qualitative defects in the rhodanese isozyme patterns of three subjects with Leber's optic neuropathy. Thus, there is no evidence for a genetic defect of a particular set of rhodanese isozymes in these subjects. However, none of the subjects was at an acute stage of the neuropathy, no subject with a reduced total hepatic rhodanese activity was studied, and in the light of the clinical heterogeneity of this disorder it would be sensible to examine liver biopsies from other case material. Unfortunately skeletal muscle and rectal mucosa biopsies used in previous studies ${ }^{89}$ were found to be unsatisfactory for electrophoretic analysis of the rhodanese isozymes.

We thank Dr M Anderson, Westminster Hospital, for performing the liver biopsies. This study was approved by the ethics committee at the Westminster Hospital.

References
1 Leber T. Ueber hereditaere und congenital angelegte Sehnerven-
leiden. A v Graefes Arch Klin Ophthmol 1871;2:249-91.
Bell J. Hereditary optic atrophy (Leber's disease). In: Treasury
of human inheritance. Vol 2 . London: Cambridge University
Press, 1933:325-423.
3 Wallace DC. Leber's optic atrophy: a possible example of
vertical transmission of a slow virus in man. Aust Ann Med
$1970 ; 19: 1-4$.
4 Erickson RF. Leber's optic atrophy, a possible example of
maternal inheritance. Am J Hum Genet $1972 ; 24: 348-9$.
5 Egger J, Wilson J. Mitochondrial inheritance in a mitochondrially
mediated disease. N Engl J Med 1983;309:142-6.
6 Wilson J. Leber's hereditary optic atrophy: a possible defect of
cyanide metabolism. Clin Sci 1965;29:505-15.
7 Cagianut B, Rhyner K, Furrer W, Schnebli HP. Thiosulphate-
sulphur transferase (rhodanese) deficiency in Leber's hereditary
optic atrophy. Lancet 1981;ii:981-2.
8 Nicoskelainen E, Hassinen IE, Paljarvi L, Lang H, Kalimo H.
Leber's hereditary optic neuroretinopathy, a mitochondrial
disease? Lancet 1984;ii:1474.
Poole CJM, Kind PRN. Deficiency of thiosulphate sulphurtrans-
ferase (rhodanese) in Leber's hereditary optic neuropathy.
Br Med J 1986;292:1229-30.
10 Whitehouse DB, Pilz AJ, Porta G, Hopkinson DA. Rhodanese isozymes in human tissues. Ann Hum Genet 1988;52:1-10.

Correspondence to $\mathrm{Dr}$ D B Whitehouse, MRC Human Biochemical Genetics Unit, The Galton Laboratory, University College London, Wolfson House, 4 Stephenson Way, London NW1 2HE. 